\title{
Analyzing the Level of Presence While Navigating in a Virtual Environment during an fMRI Scan
}

\author{
Miriam Clemente ${ }^{1, *}$, Alejandro Rodríguez ${ }^{1}$, Beatriz Rey ${ }^{1,2}$, Aina Rodríguez $^{3}$, \\ Rosa M. Baños ${ }^{2,4}$, Cristina Botella ${ }^{2,3}$, Mariano Alcañiz ${ }^{1,2}$, and César Ávila ${ }^{3}$ \\ ${ }^{1}$ Universidad Politécnica de Valencia, I3BH /LabHuman, Camino de Vera s/n, 46022, \\ Valencia (Spain) \\ ${ }^{2}$ Ciber Fisiopatología Obesidad y Nutrición (CB06/03) Instituto Salud Carlos III, Spain \\ ${ }^{3}$ Departamento de Psicología Básica, Clínica y Psicobiología.- Universidad \\ Jaume I de Castellón (España) \\ ${ }^{4}$ Departamento de Personalidad, Evaluación y Tratamientos Psicológicos- \\ Universidad de Valencia (España) \\ \{mclemente, arodriguez, brey, malcaniz\} @labhuman. i3bh.es
}

\begin{abstract}
We have conducted an fMRI research using virtual reality to study the level of presence that subjects experience during the navigation through a virtual environment, in comparison with the presence felt during a video or a photograph viewing task. The fMRI results have not been analyzed yet, but responses to presence questionnaires have been analyzed. Presence levels are similar to those obtained while monitoring with other brain imaging techniques. The highest values are obtained for navigation tasks followed by video and photographs tasks.
\end{abstract}

Keywords: fMRI, presence, virtual reality, navigation, SUS questionnaire.

\section{Introduction}

Virtual environments can evoke in the subject the feeling of "being there", commonly known as presence, despite your body is physically located in another place $[1,2]$. The traditional way of measuring presence has been using questionnaires [2, 3]. Objective techniques have also been proposed, mainly based on psychophysiological measurements, such as skin conductivity and heart rate [4]. Neurological measures such as EEG or Transcranial Doppler (TCD) have also been used for this purpose. Regarding TCD, two recent studies [5, 6] found that the major cerebral arteries showed changes in blood flow velocity associated with different levels of presence in different immersive and navigation conditions.

Functional magnetic resonance (fMRI) has been used combined with VR for different purposes. Pine et al. [7] analyzed the brain areas activated during the performance of a navigation task without explicitly measuring presence. Hoffman et al. [8] measured by questionnaires the level of presence experimented during an fMRI scan, observing that, despite the constraints of the fMRI machine, the illusion of

\footnotetext{
${ }^{*}$ Corresponding author.
} 
presence was possible. Baumgartner et al. [1] used fMRI to analyze brain activation associated to presence during a video of a virtual experience.

Our current research is trying to find the brain areas associated to the sense of presence during a virtual reality paradigm where the participants can navigate freely, in comparison with less immersive conditions, using fMRI. The main hypothesis of our research is that brain activation would be higher in a navigation task than in a video or photographs one. Although the fMRI data have not been analyzed yet, we will present the level of presence evaluated by means of a validated questionnaire [3]. These questionnaire results will be compared with those obtained in a previous work developed with TCD using the same SUS questionnaire [5].

\section{Methods}

This study has been conducted among 14 right-handed women, between 19 and 25 years old (mean $21.643 \pm 2.098$ ), all without any medical or psychological disorder. Hand dominance was tested by the Edinburgh Handedness Inventory [9].

The virtual world has been developed using GameStudio software. It consisted in a common bedroom. Each of the experimental conditions is six times repeated in a counterbalanced order, to avoid effects produced by the order of the tasks. A searching task (key counting) has been introduced to avoid that the subjects remain still during the period. The total time of the whole experiment is 12 minutes 52 seconds. Tasks were trained before the experiment. As can be observed in Figure 1, each repetition of the experimental condition (20 seconds) is preceded by a label indicating the condition and followed by a question about the key counting task.

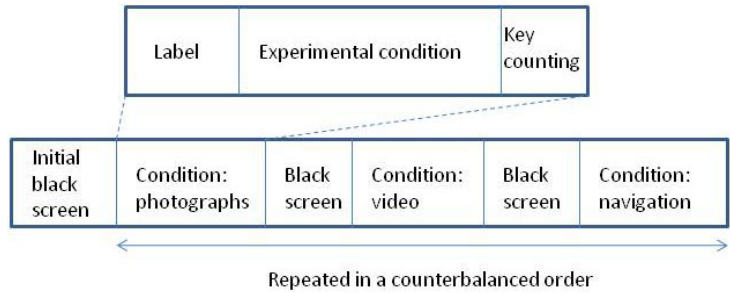

Fig. 1. Diagram of the experimental task

All subjects were scanned during exposure to the different experimental conditions in a 1.5 Teslas Siemens Avanto Magnetic Resonance scanning device (General Hospital, Castellón, Spain). For showing the environments, we used special MRI glasses, VisualStim Digital; and, for the navigation, we used an adapted joystick. First of all, sagittal T1-weighted structural images were acquired, and then the functional scanning was launched synchronized with the virtual environments. Functional images were obtained using a single-shot echo-planar imaging (EPI) sequence.

After the scan, subjects are required to answer six 7-point Likert type questions of a SUS questionnaire [3] to evaluate the level of presence that they have felt during each of the three tasks. The SUS questionnaires [3] were analyzed using SPSS 17.0. We calculated an additional measurement: SUS mean, which is the mean score across 
the six questions. We applied the nonparametric Friedman Test to compare between SUS responses (dependent variables) in the different experimental conditions (independent variable). Post-hoc tests were made with a Wilcoxon Signed-Rank test with Bonferroni correction.

The results have been compared with the SUS questionnaires responses from 9 women obtained in a similar TCD study [5] that also evaluated differences between two conditions: free and automatic navigation. In that case, the environments were visualized in a CAVE-like system. We applied a repeated measures ANOVA to evaluate the influence on the dependent variable (SUS mean) of the within-subjects factor ( navigation versus video) and the between-subjects factor (fMRI versus TCD). The homocedasticy was evaluated with the Levene statistic.

\section{Results}

We applied the non-parametric Friedman Test to the questionnaire answers, obtaining significant differences between the three experimental conditions in all the questions except question 5. The summary of the questionnaire results for each task and the Friedman results for each question can be seen in Table 1. We conducted Post-hoc analyses based on Wilcoxon Signed-Rank Tests over the SUS mean results with Bonferroni correction $(p<0.016)$. We found no significant differences between the photographs and the video tasks $(Z=-1.174, p=0.241)$. However, there was a statistically significant increment in SUS mean in the navigation vs. photographs trial $(\mathrm{Z}=-2.805, p=0.005)$ and the navigation vs. video trial $(\mathrm{Z}=-2.550, \mathrm{P}=0.011)$.

Regarding the evaluation of the influence on SUS mean of the kind of technique used for monitoring brain activation (TCD versus fMRI), we found no significant effect for this factor $(\mathrm{F}(1,21)=2.701, \mathrm{p}=0.115)$. A significant effect was found for the navigation factor $(\mathrm{F}(1,21)=11.598, \mathrm{p}=0.003<0.005)$ and no effect was found for the interaction between navigation and monitoring technique $(F(1,21)=0.751, p=0.396)$. A power analysis using the $G^{*}$ power3 program [10] showed that a total sample of 42 subjects would have been required to obtain the recommended $80 \%$ power in a t test comparison between fMRI and TCD, with alpha set at 0.05 and Cohen's d at 0.8 (large effect size).

Table 2. SUS responses (mean value and standard error of the mean) to the questionnaires and results of the Friedman Test for each question and the mean

\begin{tabular}{cccccc}
\hline Question & Photographs & Video & Navigation & $\chi^{2}$ & $\mathrm{p}$ \\
\hline Question1 & $3.1429 \pm 0.3902$ & $3.7857 \pm 0.4470$ & $4.4286 \pm 0.4286$ & 16.000 & $<0.001$ \\
Question2 & $2.7857 \pm 0.4824$ & $3.1429 \pm 0.4788$ & $3.5000 \pm 0.5000$ & 6.750 & 0.034 \\
Question3 & $2.0000 \pm 0.3145$ & $2.5000 \pm 0.4160$ & $3.1429 \pm 0.4901$ & 10.903 & 0.004 \\
Question4 & $3.1429 \pm 0.3759$ & $3.1429 \pm 0.4901$ & $4.0714 \pm 0.4505$ & 6.450 & 0.004 \\
Question5 & $3.4286 \pm 0.4412$ & $3.5000 \pm 0.4160$ & $4.0000 \pm 0.4570$ & 5.250 & 0.072 \\
Question6 & $2.7143 \pm 0.3696$ & $3.0000 \pm 0.5027$ & $3.5000 \pm 0.5320$ & 6.067 & 0.048 \\
SUSmean & $2.8693 \pm 0.3303$ & $3.1788 \pm 0.4037$ & $3.7733 \pm 0.4256$ & 12.293 & 0.002 \\
\hline
\end{tabular}




\section{Conclusions}

We have analyzed the presence questionnaires results in different experimental conditions (navigation, video and photographs) during a virtual reality scan. We have observed that the maximum experience of presence occurred during the navigation task, with lower rating for the video and minimum for the photographs. Comparing our results with those obtained in a previous research about presence using TCD, we found similar presence levels in both studies, presenting a similar trend between both tasks. In fact, we found significant differences between the conditions for both groups, but no differences between the groups were observed. There is only a trend (that does not reach significance) to higher presence ratings in the TCD research, probably due to the more immersive environment (the CAVE-like configuration) and the less intrusive machine (the TCD probes). The magnetic resonance is noisy, requires you to be still and laid and makes difficult the feeling of "being there".

Our research is the first designed using fMRI to analyze the differences in the sense of presence between navigation, video and photographs. We expect that the fMRI analysis will provide information about the brain areas that are activated during the presence experience associated to a free navigation in a virtual environment.

\section{References}

1. Baumgartner, T., Speck, D., Wettstein, D., Masnari, O., Beeli, G., Jäncke, L.: Feeling present in arousing virtual reality worlds: prefrontal brain regions differentially orchestrate presence experience in adults and children. Frontiers in Human Neuroscience 2, 1-12 (2008)

2. Baños, R.M., Botella, C., García-Palacios, A., Villa, H., Perpiñá, C., Alcañiz, M.: Presence and reality judgment in virtual environments: A unitary construct? CyberPsychology \& Behavior 3, 327-335 (2000)

3. Usoh, M., Catena, E., Arman, S., Slater, M.: Using presence questionnaires in reality. Presence: Teleoperators and Virtual Environments 9, 497-503 (2000)

4. Meehan, M., Insko, B., Whitton, M., Brooks Jr., F.P.: Physiological measures of presence in virtual environments. Paper presented at: 4th International Workshop on Presence (Philadelphia, Pennsylvania, USA) (2001)

5. Alcañiz, M., Rey, B., Tembl, J., Parkhutik, V.: A neuroscience approach to virtual reality experience using transcranial Doppler monitoring. Presence 18, 97-111 (2009)

6. Rey, B., Alcañiz, M., Tembl, J., Parkhutik, V.: Brain activity and presence: a preliminary study in different immersive conditions using transcranial Doppler monitoring. Virtual Reality 14, 55-65 (2010)

7. Pine, D.S., Grun, J., Maguire, E.A., Burgess, N., Zarahn, E., Koda, V., Fyer, A., Szeszko, P.R., Bilder, R.M.: Neurodevelopmental aspects of spatial navigation: a virtual reality fMRI study. Neuroimage 15, 396-406 (2002)

8. Hoffman, H.G., Richards, T., Coda, B., Richards, A., Sharar, S.R.: The illusion of presence in immersive virtual reality during an fMRI brain scan. CyberPsychology \& Behavior 6 , 127-131 (2003)

9. Oldfield, R.C.: The assessment and analysis of handedness: the Edinburgh inventory. Neuropsychologia 9, 97-113 (1971)

10. Faul, F., Erdfelder, E., Lang, A.-G., Buchner, A.: G*Power 3: A flexible statistical power analysis program for the social, behavioral, and biomedical sciences. Behavior Research Methods 39, 175-191 (2007) 